\title{
Feasibility of a Flipped Classroom approach in the Dietetic Program: an Italian experience
}

\author{
Maria Pilar Princis ${ }^{1}$, Rosella Bazzano ${ }^{1}$, Debora Porri ${ }^{2}$ and Hellas Cena ${ }^{1,3}$ \\ ${ }^{1}$ Department of Public Health, Experimental and Forensic Medicine, Unit of Human Nutrition, University of Pavia, \\ Pavia, Italy, \\ ${ }^{2}$ Laboratory of Dietetics and Clinical Nutrition, Department of Public Health, Experimental and Forensic Medicine, \\ University of Pavia, Pavia, Italy and \\ ${ }^{3}$ Clinical Nutrition and Dietetics Service, Unit of Internal Medicine and Endocrinology, ICS Maugeri IRCCS, \\ University of Pavia, Pavia, Italy, Pavia, Italy
}

\begin{abstract}
Alternative teaching methods focusing on learner-centered and competency-based education lead to an increase in knowledge and skills students will have to apply after graduation ${ }^{(1)}$; it has been shown that expecting to teach improves learning ${ }^{(2)}$. Flipped classroom is an instructional approach combining individual and team work with active learning strategies ${ }^{(3)}$. Its potential in nutrition courses has been formerly demonstrated, but the results focused on students' satisfaction ${ }^{(1)}$. Aim of this study was to evaluate flipped classroom efficacy for Dietetics students' achievement in dietetics and nutritional therapy. The study was conducted at University of Pavia, Italy.

Sixteen students ( 8 males) participated in flipped learning during diet therapy course in the third year. They attended 32 hours of in-class activities, case reports discussions, group-based problem solving activities facing 10 different physiological and clinical conditions implementing the Nutrition Care Process approach. Students were assessed during each lesson and with a low stake test. A mid-term anonymous questionnaire using the 5 Likert scale items was submitted in order to evaluate any critical issues besides the degree of satisfaction. Data were analyzed by frequency.

Both teamwork and individual initiative and problem solving skills were assessed: 12 students out of 16 were positively evaluated; $38 \%$ of students contributed greatly to the discussion in class (more than 5 times during the course). Students showed a high level of frequency in using scientific evidence, authoritative websites, guidelines and position papers (80\%). All improved their slideshow contribution, becoming more effective as course progressed, scoring high (7/10) at the end of the course. Students were satisfied with the flipped learning class overall (68\%), referring that flipped learning was helpful for self-directed learning (80\%) and motivation for learning $(81 \%)$, they enjoyed the use of training videos and would have liked to be able to use more $(86 \%)$.

The Flipped classroom is a valid approach when applied to final year students who already have reached autonomy in scientific literature search and identification of the appropriate sources for problem solving and clinical cases discussion. It was observed that flipped learning is helpful for the interaction and collaboration in the classroom. However conclusions are difficult to be drawn because this study was conducted only once in only one course, so it is hard to generalize the results. Besides further studies are recommended to compare an experimental group with a control group.
\end{abstract}

\section{Conflict of Interest}

There is no conflict of interest.

\section{References}

1. Gilboy et al. (2015).

2. Nestojko et al. (2014).

3. Bergmann et Sams. (2012). 